\title{
MIN-MAX ROBUST EMERGENCY SERVICE SYSTEM DESIGN
}

This paper deals with the emergency service system design using the weighted p-median problem formulation. In such systems, not only the disutility of an average user is minimized, but also the disutility of the worst situated users must be taken into account. To cope with both objectives, we suggest a composed method. In the first phase, the disutility of the worst situated user is minimized. The second phase is based on the min-sum approach to optimize the average user's disutility. To formulate the mathematical models, the radial approach is used mainly for its excellent performance characteristics. Within this paper, we concentrate on effective usage of the radial approach to develop an algorithm for robust emergency service system design. A robust service system design is usually performed so that the design complies with specified scenarios so that the maximal objective function value of the individual instances corresponding with particular scenarios is minimized. To find the value paid for making the system resistant to catastrophic events, a new conception called the price of robustness is introduced.

Keywords: Emergency service system, min-max approach, radial formulation, robustness.

\section{Introduction}

Emergency service system design for a given road network in serviced area locates limited number of service centers at positions from a given set of possible locations to satisfy future system users' demands for service in case of emergency [1] Different objectives can be applied on the design. A traditional one is minimal disutility perceived by an average user. In such a case, the perceived disutility is assumed to be proportional to the distance of a user location from the nearest located service center and then, sum of distances from particular system users to the nearest located service center is minimized. Such objective is referred to as min-sum criterion and is broadly used in private service system designs, where service delivering is provided and paid by system owner. In contrast to the private systems, users of an emergency public service system share cost of the system by paying tax, which approves them to claim the equal or fair access to the provided service. In general, the fairness emerges whenever limited resources are to be fairly distributed among participants [2, 3 and 4]. Plethora of fairness schemes were studied, but the strongest one applicable in the public service system design is so called lexicographic min-max criterion [5, 6, and 7]. As the lexicographical min-max approach produces such a system design, where the price of fairness, i.e. relative deterioration of the average user's disutility, is too high, composed approaches were designed to mitigate this drawback [8]. A composed approach usually performs two phases, where the first one strives to minimize disutility perceived by the worst situated users using min-max objective and the second phase applies the min-sum approach under condition that the disutility of the most exposed user must not be worsened.

As traversing time between service center and an affected user might be impacted by various random events following weather or traffic, the system designer must face the demand for system resistance to such critical events [9 and 10]. Most of the approaches to increasing the system resistance are based on making its design resistant to possible failure scenarios, which can appear in the road network as a consequence of random failures due to congestion, disruptions or blockages. An individual scenario is characterized by particular time distances between the users' and possible service center locations. A robust service system design has to comply with all the specified scenarios. The usual way of taking into account all scenarios is based on minimizing the maximal objective function of the individual instances corresponding with the particular scenarios. The min-max link-up constraints represent an undesirable burden in any integer programming problem due to bad convergence of the branch-and-bound method, which dominates solving tools of available IP-solvers. Thus these approaches to the robustness constitute a big challenge to family of operational researchers and professionals in informatics.

\footnotetext{
* Jaroslav Janacek, Marek Kvet

Faculty of Management Science and Informatics,University of Zilina, Slovakia

University Science Park, University of Zilina, Slovakia

E-mail: jaroslav.janacek@fri.uniza.sk
} 
Within this paper, we focus on the emergency service system design, which is robust considering given finite set of scenarios. The basic design problem is formulated as min-max first and min-sum second composed approach, which means that the accented objective is to minimize disutility perceived by the worst situated user and the point of the average user is of secondary importance. The basic design is computed for the original scenario, which corresponds to the usual situation in the network, i.e. situation, which does not correspond to any of the above-mentioned possible failure scenarios.

Complexity of location problems with limited number of facilities to be deployed and the necessity to solve large instances of the problem led to searching for a suitable algorithm. It was found that in contrast to original location-allocation formulation, the radial formulation of the problem can considerably accelerate the associated solving process [11 and 12]. Simultaneously, an attention was paid to the radial formulation with homogenous system of radii [13]. As this approximate approach used for the "system optimal" public service system design proved to be a suitable and enough precise tool, we decided to apply the radial formulation with homogenous system of radii also on the robust emergency system design.

The remainder of the paper is organized as follows: Section 2 is devoted to the description of original composition of robust min-max and min-sum method including the radial formulation. The customization of the suggested method is described in Section 3 and the associated numerical experiments are performed in Section 4. The results and findings are summarized in Section 5.

\section{Min-max optimal robust design of emergency service system}

The min-max public service system design problem can be described by the following denotation. Let symbol $J$ denote the set of users' locations and symbol $I$ denote set of possible service center locations. We denote by $b$ the number of users, who share the location $j$. To solve the problem, at most $p$ locations must be chosen from $I$ so that the maximal disutility perceived by the worst situated user be minimal. The value of user's disutility is given by the mutual positions of the users' location and the location of the service center providing them with service. Let symbol $U$ denote the set of possible failure scenarios. We assume that user's disutility grows with increasing distance between the user and the service center. Disutility following from the distance between locations $i$ and $j$ under a specific scenario $u \in$ $U$ is denoted as $d_{i j u}$. The decisions, which determine the designed public service system, can be modeled by further introduced decision variables. The variable $y_{i} \in\{0,1\}$ models the decision on service center location at place $i \in I$. The variable takes the value of 1 if a service center is located at $i$ and it takes the value of 0 otherwise.
We use also the variable $h_{u}$ as the upper bound of the all perceived disutility values under scenario $u \in U$. To obtain an upper or a lower bound of the original objective function, the range $\left[d_{0}, d_{m}\right]$ of all $m+1$ possible disutility values $d_{0}<d_{1}<\ldots<d_{m}$ from the matrix $\left\{d_{i j u}\right\}$ is partitioned into $v+1$ zones according to [13 and 14]. The zones are separated by values from a finite ascending sequence of so called dividing points $D_{l}, D_{2} \ldots D_{v}$ chosen from the sequence $d_{0}<d_{1}<\ldots<d_{m}$, where $0=d_{0}=D_{0}<D_{1}$ and also $D_{v}<D_{v+1}=d_{m}$. The zone $s$ corresponds to the right-closed interval $\left(D_{s}, D_{s+1}\right]$. The length of the $s$-th interval is denoted by $e_{s}$ for $s=0$ ... v. Further, auxiliary zero-one variables $x_{j u s}$ for $j \in J, u \in U$ and $s=0, \ldots, v$ are introduced. The variable $x_{j u s}$ takes the value of 1 , if the disutility of the user located at $j \in J$ under scenario $u \in U$ from the nearest located center is greater than $D_{s}$ and it takes the value of 0 otherwise. Then the expression $e_{0} x_{j u 0}+e_{T} x_{j u 1}+e_{z} x_{j u 2}$ $+\ldots+e_{v} x_{j u v}$ constitutes an upper approximation of the disutility $d_{j u}^{*}$ perceived at user location $j$ from the nearest located service center under scenario $u \in U$. If the disutility $d^{*}{ }_{j u}$ belongs to the interval $\left(D_{s}, D_{s+1}\right]$, then the value of $D_{s+1}$ is the upper estimation of $d^{*}{ }_{j u}$ with the maximal possible deviation $e_{s}$. Let us introduce a zero-one constant $a_{i j u}{ }^{s}$ under scenario $u \in U$ for each triple $[i, j, s]$, where $i \in I, j \in J, s=0, \ldots, v$. The constant $a_{i j u}{ }^{s}$ is equal to 1 , if the disutility $d_{i j u}$ perceived at the user location $j$ from the possible center location $i$ is less or equal to $D_{s}$, otherwise $a_{i j u}{ }^{s}$ is equal to 0 . Then the radial-type min-max public service system design problem under given scenario $u \in U$ can be formulated as follows:

Minimize $h_{u}$

Subjet to: $x_{j s u}+\sum_{i \in I} a_{i j u}^{s} y_{i} \geq 1$

for $j \in J, s=0,1, \ldots, v$

$\sum_{i \in I} y_{i} \leq p$

$\sum_{s=0}^{v} e_{s} x_{j u s} \leq h_{u}$ for $j \in J$

$y_{i} \in\{0,1\}$ for $i \in I$

$x_{j u s} \geq 0$ for $j \in J, s=0,1, \ldots, v$

$h_{u} \geq 0$

In this model, the objective function (1) represented by single variable $h_{u}$ gives the upper bound of the all perceived disutility values. The constraints (2) ensure that the variables $x_{j u s}$ are allowed to take the value of 0 , if there is at least one center located in radius $D_{s}$ from the user location $j$ and constraint (3) limits the number of located centers by $p$. The link-up constraints (4) ensure that each perceived disutility is less than or equal to the upper bound $h_{u}$. 
As concerns the obligatory constraints (6), only values zero and one are expected in a feasible solution, but it can be seen that the model has integrality property regarding the variables $x_{j u s}$. It can be noticed that in the optimization process all relevant values of $x_{j u s}$ are "pushed down" and the constraints (2) and (6) bound the variable $x_{\text {jus }}$ from below by value of one or zero. It follows that the relevant values of $x_{\text {jus }}$ stay at one of these values.

The above-described way of modeling the min-max problem will be called "standard" min-max approach in the remainder of the paper.

Having solved the above problem, we denote $h_{u}{ }^{*}$ the optimal value of the objective function (1), then we can formulate the second phase of the composed approach to the design problem under the given scenario $u$ by the following model:

Minimize $\sum_{j \in J} b_{j} \sum_{s=0}^{v} e_{s} x_{j u s}$

Subject to: $\sum_{s=0}^{v} e_{s} x_{j u s} \leq h_{u}^{*}$ for $j \in J$

and (2), (3), (5), (6).

Let us denote the set of all feasible solutions $(\boldsymbol{x}, \boldsymbol{y})$ of the constraint system (2), (3), (5), (6) by $Q_{u}$, then the first phase of the robust approach for the set $U$ of scenarios can be modeled according to [9 and 10] as:

Minimize $h$

Subject to: $\sum_{s=0}^{v} e_{s} x_{j u s} \leq h$ for $j \in J, u \in U$

$\left(x_{u}, y\right) \in Q_{u}$ for $u \in U$

$h \geq 0$

The second phase of the approach to the robust emergency system design can be performed in several ways due to the point of the average user is of secondary importance. We will study here the simplified approach, which consists in minimization of the average user disutility, when each combination user location and scenario is taken into account. This approach makes use of the optimal value $h^{*}$ of (10) subject to (11)-(13) and the associated model can be stated in the following form.

Minimize $\sum_{u \in U} \sum_{j \in J} b_{j} \sum_{s=0}^{v} e_{s} x_{j u s}$

Subject to: $\sum_{s=0}^{v} e_{s} x_{j u s} \leq h^{*}$ for $j \in J, u \in U$

$\left(x_{u}, y\right) \in Q_{u}$ for $u \in U$

\section{Bisection radial approach to design of robust emergency service system}

The bisection radial approach makes use of the radial model, but it uses only its reduced form to find whether there is any solution with the objective function value less than or equal to a prescribed disutility value $D$. The bisection is performed according to subscript $s$ from the subscript range from 0 to $v$ with the goal to determine the lowest subscript, for which the objective function (17) equals to 0 .

In the following model, the zero-one variables $y_{i} \in\{0,1\}$ for $i \in I$ are also used to the decisions on locating or not a service center at the location $i$. Next, the variables $x_{j u}$ are introduced to indicate, whether user's disutility at location $j \in J$ following from the nearest located center under scenario $u \in U$ is greater than $D_{s}$. If user's disutility at location $j \in J$ under scenario $u \in U$ is greater than $D_{s}$, then the variable $x_{j u}$ takes the value of 1 , and it takes the value of 0 otherwise. The corresponding model can be formulated as follows.

Minimize $\sum_{u \in U} \sum_{j \in J} x_{j u}$

Subject to: $x_{j u}+\sum_{i \in I} a_{i j u}^{s} y_{i} \geq 1$ for $j \in J, u \in U$

$\sum_{i \in I} y_{i} \leq p$

$y_{i} \in\{0,1\}$ for $i \in I$

$x_{j u} \geq 0$ for $j \in J, u \in U$

In this model, the objective function (17) represents the number of user locations, where the perceived disutility is greater than $D_{s}$ under scenario $u \in U$. The constraints (18) ensure that the variables $x_{j u}$ are allowed to take the value of 0 , if there is at least one center located in radius $D_{s}$ from the user location $j$ under scenario $u \in U$ and constraint (19) limits the number of located service centers by $p$.

Having performed the above bisection process, we denote $s^{*}$ the minimal value of the subscript $s$, for which the objective function (17) equals to zero, we can formulate the second phase of the composed approach by the following model:

Minimize $\sum_{u \in U} \sum_{j \in J} b_{j} \sum_{s=0}^{s^{*}-1} e_{s} x_{j u s}$

Subject to: $x_{j u s}+\sum_{i \in I} a_{i j u}^{s} y_{i} \geq 1$

for $j \in J, s=0, \ldots, s^{*}-1, u \in U$

$\sum_{i \in I} a_{i j u}^{s^{*}} y_{i} \geq 1$ for $j \in J, u \in U$

$\sum_{i \in I} y_{i} \leq p$ 
$y_{i} \in\{0,1\}$ for $i \in I$

$x_{j u s} \geq 0$ for $j \in J, s=0, \ldots, s^{*}-1, u \in U$

\section{Numerical experiments}

To compare studied approaches based on the radial formulation and their usage for basic and robust design of the emergency service system, we performed the series of numerical experiments. To solve the problems described in the previous sections, the optimization software FICO Xpress 7.9 (64-bit, release 2015) was used and the experiments were run on a PC equipped with the Intel ${ }^{\circledR}$ Core $^{\mathrm{TM}}$ i7 $5500 \mathrm{U}$ processor with the parameters: $2.4 \mathrm{GHz}$ and $16 \mathrm{~GB}$ RAM.

The used benchmarks were derived from the real emergency health care system, which was originally implemented in eight regions of the Slovak Republic. For each self-governing region, i.e. Bratislava (BA), Banska Bystrica (BB), Košice (KE), Nitra (NR), Presov (PO), Trencin (TN), Trnava (TT) and Zilina (ZA), all cities and villages with corresponding number of inhabitants $b$, were taken. The coefficients $b$, were rounded to hundreds. These sub-systems cover demands of all communities - towns and villages spread over the particular regions by a given number of ambulance vehicles. In the benchmarks, the set of communities represents both the set $J$ of users' locations and also the set $I$ of possible center locations. The cardinalities of these sets vary from 87 to 664 according to the considered region. The number $p$ of located centers was derived from the original design and it varies from 9 to 67. The network distance from a user to the nearest located center was taken as an individual user's disutility.

The first set of numerical experiments was performed to compare the standard and bisection radial approaches to the emergency service system design. This comparison was performed for the basic situations (basic scenarios) in all eight self-governing regions. The obtained results are summarized in Table 1. Studied parameters of the experiments were:

CT - computational time in seconds, which was recorded for the individual phases

$h$-maximal disutility perceived by the worst situated users minSum - min-sum objective function value according to (8) for the basic scenarios of the individual self-governing regions.

Since convergence of the standard optimization process is very slow and the demanded computational time exceeds acceptable limit, the process was prematurely terminated after 1 hour. In this case, the standard approach did not reach the optimal solution, but only a near-to-optimal one. To estimate the possible difference between the obtained value of the maximal disutility perceived by the worst situated users and the optimal one, the associated lower bound LB on the optimal solution is presented.

The achieved results reported in Table 1 show that the bisection radial approach enables to overcome the weakness of the standard approach as regards the computational time and the min-max objective as well. In addition, the bisection approach yields exact min-max solution contrary to prematurely terminated standard approach. It must be noted that the min-sum objective is a secondary indicator, which does not enter the above comparison of min-max approaches. Therefore, the bisection approach was used in the following experiments aimed at the robust emergency service system design.

As far as the robust design is concerned, various scenarios for each solved instance must be defined. Due to the lack of common benchmarks for study of robustness, the scenarios used in our computational study were created in the following way. We chose 25 percent of matrix rows so that these rows correspond to the biggest cities concerning the number of users. Then we chose randomly from 5 to 15 rows and the associated disutility values in the individual rows were multiplied by the randomly chosen constant from the range 2, 3 and 4 . This way, 20 different

Comparison of the standard and bisection approach to emergency service system design applied on basic scenarios

Table 1

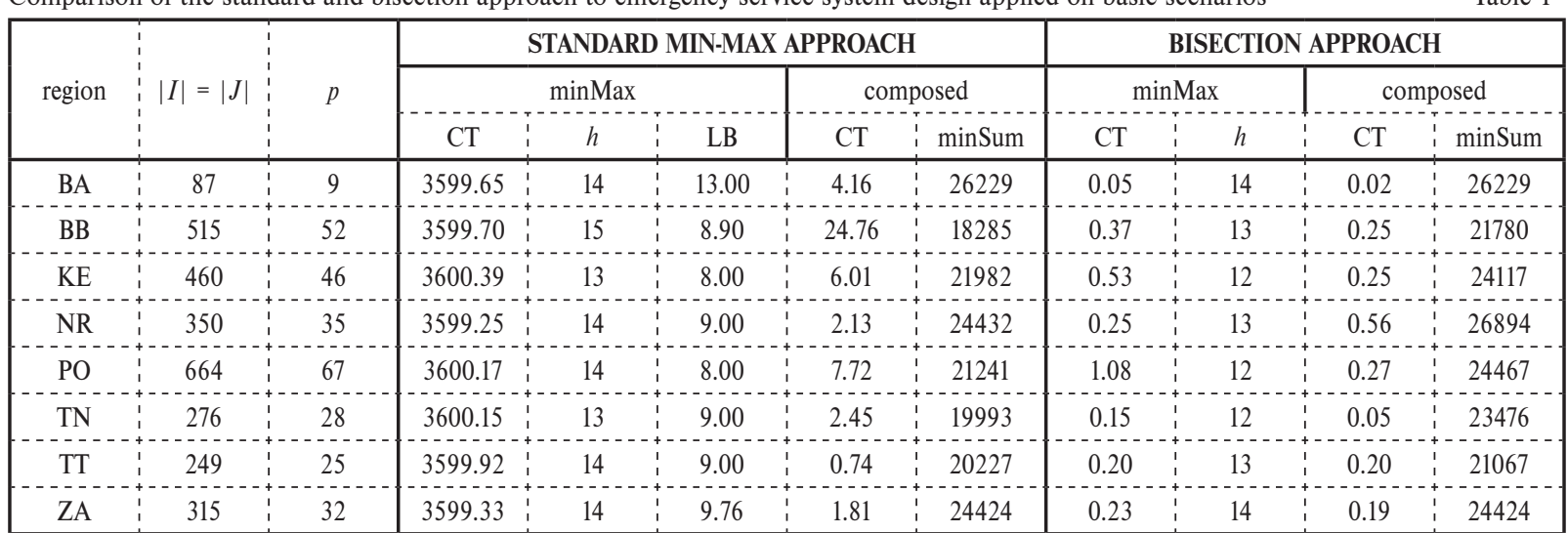


scenarios were generated for each self-governing region. It must be noted that the robust design was computed also for the whole road network of Slovakia, but only 10 scenarios were generated in this case due to the size of disutility matrix.

The comparison of the basic and robust designs of emergency service system for all self-governing regions of Slovakia is reported in Table 2. Table 3 contains the results obtained for the selfgoverning region of Zilina, where different values of parameter $p$ were considered. The parameter $p$ limits the number of service centers to be located. Both tables follow the same notation as used in Table 1. As above, CT denotes the computational time in seconds. It must be noted that the reported value contains the computational time of both phases, i.e. min-max and min-sum optimization processes. The maximal disutility perceived by the worst situated users is denoted by $h_{b}$ for the basic design and the symbol $h_{r}$ is used for the robust design. The associated value of the min-sum objective function ( 8 ) is given in the columns denoted by $m S_{b}$ and $m S_{r}$ respectively.

An individual experiment was organized so that the basic design was computed first. This way, the values $h_{b}$ and $m S_{b}$ were obtained. The resulting design, especially the values of location variables $y_{i}$ were applied on all generated scenarios and for each scenario the maximal disutility perceived by the worst situated users was computed. The worst (highest) maximal disutility value out of all scenarios is denoted by $w h$. The associated value of the min-sum objective function (8) is given in the column denoted by $w m S$.

After the basic approach was computed and applied on all scenarios, the robust problems were solved. Here, the computational time is several times bigger, because the models of the solved problems contain much more variables and structural constraints as well (all scenarios are taken into account simultaneously). The resulting vector of location variables $y_{i}$ was substituted into the objective functions (primary and secondary) associated with the basic scenario and the maximal disutility perceived by the worst situated users as well as the associated min-sum objective function were computed. These values are denoted by $h_{r}$ and $m S_{r}$ respectively. Similarly to the basic approach, the worst (highest) maximal disutility value out of all scenarios is reported in the column denoted by $w h$. The associated value of the min-sum objective function (8) is given in the column $w m S$.

The basic and robust approaches to the emergency service system design were compared from two points of view. First, the vectors of location variables $y_{i}$ were compared by the Hamming distance HD, which is defined as follows. Let $y^{b}$ denote the vector

Results of numerical experiments comparing the basic and robust design of emergency service system for the self-governing regions of Slovakia

Table 2

\begin{tabular}{|c|c|c|c|c|c|c|c|c|c|c|c|c|c|c|c|}
\hline \multirow{2}{*}{  } & \multirow{2}{*}{$|I|=|J|$} & \multirow[b]{2}{*}{$p$} & \multicolumn{5}{|c|}{ BASIC DESIGN } & \multicolumn{8}{|c|}{ ROBUST DESIGN } \\
\hline & & & $\mathrm{CT}$ & $h_{b}$ & $m S_{b}$ & wh & $w m S$ & CT & $h_{r}$ & $m S_{r}$ & wh & $w m S$ & HD & $\mathrm{PoR}_{1}$ & $\mathrm{PoR}_{2}$ \\
\hline $\mathrm{BA}$ & 87 & 9 & 0.07 & 14 & 26229 & 41 & 41201 & 1.24 & 15 & 53567 & 15 & 53567 & 14 & 7.14 & 104.23 \\
\hline $\mathrm{BB}$ & 515 & 52 & 0.62 & 13 & 21780 & 28 & 22640 & 17.69 & 14 & 26890 & 14 & 27867 & 68 & 7.69 & 23.46 \\
\hline KE & 460 & 46 & 0.78 & 12 & 24117 & 26 & 27864 & 51.67 & 13 & 28391 & 13 & 30076 & 52 & 8.33 & 17.72 \\
\hline NR & 350 & 35 & 0.81 & 13 & 26894 & 28 & 29497 & 14.29 & 14 & 31584 & 14 & 31994 & 50 & 7.69 & 17.44 \\
\hline $\mathrm{PO}$ & 664 & 67 & 1.34 & 12 & 24467 & 29 & 24901 & 35.19 & 13 & 29137 & 13 & 30365 & 78 & 8.33 & 19.09 \\
\hline TN & 276 & 28 & 0.20 & 12 & 23476 & 24 & 27496 & 6.85 & 14 & 23489 & 14 & 24000 & 40 & 16.67 & 0.06 \\
\hline $\mathrm{TT}$ & 249 & 25 & $0.40^{-}$ & 13 & 21067 & 23 & 23931 & 4.10 & 13 & 30332 & 13 & 30783 & 44 & 0.00 & 43.98 \\
\hline $\mathrm{ZA}$ & 315 & 32 & 0.42 & 14 & 24424 & 29 & 30859 & 6.02 & 15 & 26517 & 15 & 27758 & 34 & 7.14 & 8.57 \\
\hline SR & 2916 & 273 & 47.07 & 13 & 193715 & 22 & 194599 & 573.22 & 13 & 203988 & 13 & 204450 & 86 & 0.00 & 5.30 \\
\hline
\end{tabular}

Results of numerical experiments comparing the basic and robust design of emergency service system for the self-governing region of Zilina and different numbers of located service centers

Table 3

\begin{tabular}{|c|c|c|c|c|c|c|c|c|c|c|c|c|c|c|}
\hline \multirow{2}{*}{$|I|=|J|$} & \multirow[b]{2}{*}{$p$} & \multicolumn{5}{|c|}{ BASIC DESIGN } & \multicolumn{8}{|c|}{ ROBUST DESIGN } \\
\hline & & $\mathrm{CT}$ & $h_{b}$ & $m S_{b}$ & wh & $w m S$ & $\mathrm{CT}$ & $h_{r}$ & $m S_{r}$ & wh & $w m S$ & HD & $\mathrm{PoR}_{1}$ & $\mathrm{PoR}_{2}$ \\
\hline 315 & 158 & 0.10 & 4 & 3213 & 12 & 3446 & 4.12 & 4 & 4329 & 4 & 4389 & 50 & 0.00 & 34.73 \\
\hline 315 & 105 & 0.21 & 6 & 6512 & 14 & 6882 & 4.28 & 6 & 10539 & 6 & 10842 & 58 & 0.00 & 61.84 \\
\hline 315 & 79 & 0.21 & 7 & 12428 & 20 & 13637 & 4.41 & 8 & 15090 & 8 & 15339 & 92 & 14.29 & 21.42 \\
\hline 315 & 63 & 0.35 & 8 & 20960 & 23 & 22996 & 5.05 & 10 & 15297 & 10 & 15873 & 74 & 25.00 & 27.02 \\
\hline 315 & 32 & 0.44 & 14 & 24424 & 36 & 30113 & 5.56 & 15 & 29522 & 15 & 30666 & 42 & 7.14 & 20.87 \\
\hline 315 & 21 & 0.44 & 16 & 37887 & 36 & 43540 & 7.13 & 18 & 44550 & 18 & 46190 & 34 & 12.50 & 17.59 \\
\hline 315 & 16 & 0.50 & 20 & 46647 & 42 & 49636 & 8.67 & 21 & 53884 & 21 & 53884 & 26 & 5.00 & 15.51 \\
\hline
\end{tabular}


of location variables for the basic design and let $\boldsymbol{y}^{\prime \prime}$ denote the vector for the robust one. Then the Hamming distance HD takes the form of (28).

$$
H D=\sum_{i \in I}\left(y_{i}^{r}-y_{i}^{b}\right)^{2}
$$

Hamming distance evaluates the structural difference between two designs in the sense that it informs of number of locations, in which the designs differ, but it does not refer to the quality of the designs. Therefore, we have compared the basic and robust design also from the viewpoint of other characteristics. Similarly to the price of fairness introduced and studied in [4 and 14] to evaluate the loss of min-sum objective function value caused by application of measures for fairness improvement, we introduce here so-called price of robustness. The primary price of robustness $P_{0} R_{l}$ expresses the difference between the maximal disutility values perceived by the worst situated users in the solutions obtained by the basic and robust approaches applied on the basic scenario. The value of $P o R_{l}$ is given in percentage and it can be computed according to $(29)$.

$$
P o R_{1}=100 * \frac{h_{r}-h_{b}}{h_{b}}
$$

The secondary price of robustness $P_{0} R_{2}(30)$ is similar to the primary one, but it takes into account the min-sum objective function values $m S_{b}$ and $m S_{r}$.

$$
P_{o R_{2}}=100 * \frac{m S_{r}-m S_{b}}{m S_{b}}
$$

As concerns the comparison performed in Tables 2 and 3, it must be noted that the primal criterion in the designed emergency systems is the maximal disutility perceived by the worst situated user. It means that the primary objective of the robust system design should be resistant to changing scenarios as much as possible. Therefore, the values of $w h$ in the basic and robust designs give the substantial information for the comparison. The value $w h$ gives the highest maximal disutility out of all scenarios.

\section{Conclusions}

We have suggested and verified a useful tool for robust design of emergency service system. The suggested tool is able to comply with the problem which size is several times bigger than the size of the standard emergency system design problem. The bigger size of the robust problem is caused by cardinality of the set of studied scenarios. Good time performance of suggested approach follows from smart bisection process applied in the first phase of the suggested algorithm. Usage of the radial approach proved its usefulness especially in the second phase, where the min-sum problem is solved. As can be noticed, the computational time stays acceptable even if the size of the problem is several times swollen. To be able to answer the question what we have paid for the robustness of the designed system, we have introduced the price of robustness. The primary price of robustness expresses the relative difference between the maximal disutility values perceived by the worst situated users in the standard solution and the robust solution. The robustness measure has similar meaning as the price of fairness commonly used to evaluate the loss of min-sum objective function value caused by application of measures for fairness improvement. The price of robustness might help the designer to find the value paid for making the system resistant to catastrophic events.

The future research in this field may be aimed at finding relevant scenarios, which can significantly impact the performance of emergency service system. In connection with possible high price of robustness, it can be valuable to focus future research on development of such compromising approach, which can limit the price of robustness not to spoil the standard solution too much. From the practical point of view, we will try to find such method, which allows to change only limited number of service center locations in comparison with the standard solution. This algorithm may be useful when current service system is subjected to a reengineering process and stability of current service center deployment is required.

\section{Acknowledgement}

This work was supported by the research grants VEGA 1/0518/15 "Resilient rescue systems with uncertain accessibility of service", VEGA 1/0463/16 "Economically efficient charging infrastructure deployment for electric vehicles in smart cities and communities", APVV-15-0179 "Reliability of emergency systems on infrastructure with uncertain functionality of critical elements" and by the project University Science Park of the University of Žilina (ITMS: 26220220184) supported by the Research \& Development Operational Program funded by the European Regional Development Fund. We would also like to thank to "Centre of excellence for systems and services of intelligent transport" (ITMS 26220120050) for built up the infrastructure, which was used.


Európska únia is -1 is

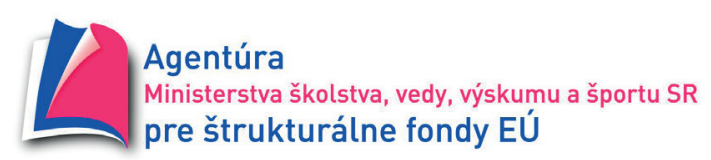




\section{References}

[1] JANOSIKOVA, L.: Emergency Medical Service Planning. Communications - Scientific Letters of the University of Zilina, ISSN 13354205, vol. 9, No. 2, pp. 64-68, 2007.

[2] NASH, J.: The Bargaining Problem. Econometrica, vol. 18, No. 2, pp. 155-162, 1950.

[3] MARSH, M., SCHILLING, D.: Equity Measurement in Facility Location Analysis. European J. of Operational Research, 74, pp. 1-17, 1994.

[4] BERTSIMAS, D., FARIAS, V. F., TRICHAKIS, N.: The Price of Fairness. Oper. Res., 59, pp. 17-31, 2011.

[5] OGRYCZAK, W., SLIWINSKI, T.: On Direct Methods for Lexicographic Min-Max Optimization. In: Gavrilova M. et al. (Eds.): ICCSA 2006, LNCS 3982, (pp. 802-811). Berlin: Heidelberg: Springer, 2006.

[6] BUZNA, L., KOHANI, M., JANACEK, J.: Proportionally Fairer Public Service Systems Design. Communications - Scientific Letters of the University of Zilina, ISSN 1335-4205, vol. 15, No. 1, pp. 14-18, 2013.

[7] CEBECAUER, M., BUZNA, L.: Re-Aggregation Heuristics for the Large Location Problems with Lexicographic Minimax Objective. Communications - Scientific Letters of the University of Zilina, ISSN 1335-4205, vol. 17, No. 2, pp. 4-10, 2015.

[8] JANACEK, J., KVET, M.: Emergency System Design with Temporarily Failing Centers. SOR 15: Proc. of the $13^{\text {th }}$ Intern. Symposium on Operational Research, Ljubljana: Slovenian Society Informatika: Section for Operational Research, ISBN 978-961-6165-45-7, pp. 490-495, 2015.

[9] PAN, Y., DU, Y., WEI, Z.: Reliable Facility System Design Subject to Edge Failures. American J. of Operations Research, 4, pp. 164-172, 2014.

[10] CORREIA, I., SALDANHA da GAMA, F.: Facility Locations under Uncertainty. Location Science, eds. Laporte: Nikel, Saldanha da Gama, pp. 177-203, 2015.

[11] GARCIA, S., LABBE, M., MARIN, A.: Solving Large p-median Problems with a Radius Formulation. INFORMS - J. on Computing, vol. 23, No. 4, pp. 546-556, 2011.

[12] JANACEK, J.: Approximate Covering Models of Location Problems. Lecture Notes in Management Science: Proc. of $1^{\text {st }}$ Intern. Conference ICAOR '08, vol. 1, Sept. 2008, Yerevan, pp. 53-61, 2008.

[13] JANACEK, J., KVET, M.: Relevant Network Distances for Approximate Approach to the p-median Problem. Operations Research Proceedings 2012: Selected Papers of the Intern. Conference of the German operations research society (GOR), September 2012, Leibniz: Univesitat Hannover : Springer, pp. 123-128, 2014.

[14] KVET, M., JANACEK, J.: Price of Fairness in Public Service System Design. Mathematical Methods in Economics 2014, Olomouc, ISBN 978-80-244-4209-9, pp. 554-559, September 2014. 